\title{
L'humanisme dissident des rhétoriqueurs : le cas de Guillaume Cretin
}

\section{LUC \\ VAILLANCOURT}

Summary: It is not because he was unacquainted with Italian humanism that Guillaume Cretin, one of the "grands Rhétoriqueurs," a group of late-fifteenth and early-sixteenth century writers associated with the courts of France and Burgundy, chose to cultivate traditional genres and themes. His epistles reveal an excellent knowledge of the new rhetorical values, most of which he exploits himself, in rather a contrast with his usual style. Of course, Cretin is a courtier and, as such, he has a political role to playdefending his king, praising his people and cultural heritage - but this is not only a matter of propaganda, since a very coherent vision arises from his work, a vision to which he seems thoroughly committed.

$\mathrm{O}^{\prime \prime}$ sait ce que les épistoliers français de la Renaissance doivent aux humanistes italiens ${ }^{1}$. Du cicéronianisme au pétrarquisme, en passant par un certain maniérisme courtisan, l'Italie a imposé des modèles de prose néo-latine, suscité l'essor d'un sentimentalisme plaintif dans les lettres amoureuses ${ }^{2}$, informé les échanges de civilités et «contaminé » durablement le lexique de la jeune langue française. L'hégémonie culturelle semble totale de prime abord, tant sur le plan théorique, avec tous les manuels épistolaires, protocoles et secrétaires qui se contentent de reconduire les enseignements des chanceliers italiens du $\mathrm{XV}^{\mathrm{e}}$ siècle, qu'au niveau de la pratique, alors que les émules de Cicéron se multiplient suivant l'exemple de Christophe de Longueil, et que les traductions des Politien, Bembo, l'Arétin et consorts l'emportent sur les compositions originales, jusque dans les Lettres Missives et Familières d'Étienne du Tronchet (1569), qu' on serait pourtant en droit de croire de sa plume puisqu'elles portent sa signature, mais qui sont pour moitié d'origine italienne. Cette déferlante qui s'abat en raz-de-marée sur toute l'Europe n'a-t-elle rien épargné sur son passage ? 
Existe-t-il une pratique épistolaire, une topique, une forme que l'on puisse qualifier de typiquement française, susceptible peut-être (pourquoi pas ?) d'avoir contribué au dépassement de cette suprématie ?

Bien avant que Politien et l'Arétin publient leurs lettres dans leur langue natale, la France s'était illustrée par une abondante production d'épîtres vernaculaires. Au Moyen Âge, des rondeaux, des ballades et des prosimètres portent déjà le nom d'épîtres. L'œuvre de Christine de Pisan revêt volontiers cette forme, comme c'est le cas avec L'Epistre de prison de vie humaine, L'Epistre du Dieu d'Amours, L'Epistre d'Othea, en plus des autres lettres insérées dans les traités comme Le livre de la Paix ou Le livre des Trois Vertus. L'auteur puise son inspiration aussi bien chez Ovide, Sénèque, Boèce que dans la Bible et les écrits des Pères, et l'on pourrait être tenté d'assimiler sa pratique du genre, de même que celle de ses contemporains, au mouvement humaniste naissant. À la fin du XVe siècle, la traduction par Octovien de Saint-Gelais des Hérö̈des d'Ovide contribue à la vogue de l'épître et introduit un recours à la prosopopée qui sera souvent exploité par la suite. Ces lettres fictives de personnages illustres deviennent bientôt prétextes à exercices scolaires et connaissent toutes sortes d'avatars, tels l'Epistre envoyée des Champs Elisées au roy Henry d'Engleterre, à présent régnant au dit royaulme de Jean Bouchet, les deux Epistres de l'amant vert de Jean Lemaire de Belge (1505 et 1511) imputées à un perroquet décédé, ou les épîtres de "personnages historiques » insérées dans la Chronique françoyse de Guillaume Cretin. Si les œuvres des anciens font l'objet de nombreuses références, rien ne prouve cependant une médiation italienne dans le développement du genre. Au contraire, les Rhétoriqueurs auront beau fréquenter les cercles humanistes, y prendre part, vénérer, pasticher et traduire les auteurs latins, force est de constater chez eux une identification générale aux formes et aux traditions françaises plutôt qu'aux canons italiens, notamment en ce qui a trait à l'épître en vers, investie d'une dignité particulière ${ }^{3}$. On pourrait certes invoquer, on l'a déjà fait, un retard culturel, mais comment justifier son prolongement jusqu' au milieu du XVIe siècle ? Il semble plutôt qu'au contact de l'humanisme italien, une partie de l'élite française (les lettrés) a d'abord opposé une résistance et qu'elle a tenté de se définir à travers des pratiques distinctes de même que par la valorisation du vernaculaire 4 . Ainsi «Villon, Cretin ont Paris decoré » écrit Clément $\operatorname{Marot}^{5}$, suggérant que l'écrivain participe à l'illustration d'une nation. Ainsi, Jean Lemaire de Belges peut conclure, dans sa Concorde des deux langages (1511), à la supériorité morale de la poésie française. Ainsi les Rhétoriqueurs vont cultiver l'épître à « la françoyse » sans pour autant méconnaître ou renier les valeurs humanistes. 
Le cas de Guillaume Cretin en est un d'école : on lui connaît une abondante production d'épîtres en vers, disséminées à travers nombre de manuscrits, éditions collectives et individuelles, le tout rassemblé avantageusement dans l'édition critique par Kathleen Chesney de ses Euvres poétiques $^{6}$, qui nous semblent représentatives, pour le fond comme pour la forme, de la pratique française en ce début de $\mathrm{XVI}^{\mathrm{e}}$ siècle. On y retrouve des lettres fictives, telle cette épître à François I ${ }^{\mathrm{er}}$ de la part de la Chapelle de Vincennes, et ces autres au nom de Charles le Téméraire ou des Dames de Paris, mais on y relève principalement des missives présentées comme « réelles », adressées à ses protecteurs, confrères et amis. Cretin entretient des liens étroits avec les autres Rhétoriqueurs et les échanges de compliments constituent l'un des motifs les plus usités. Ces éloges mutuels, malgré leur caractère hyperbolique et artificiel, témoignent d'une foi certaine dans les mérites de chacun et, par association, contribuent à la valorisation de la poésie française. Ainsi, dans une épître À son amy Le Maire, que ce dernier va placer en guise de caution au début de son Temple d'Honneur et de Vertus (1503), Cretin laisse libre cours à l'expression de son enthousiasme :
Se le escriptz tant modernes que antiques
Par motz dorez et azurez cantiques
Ont mérité prendre sur terre nom
De riche loz et glorieux renom,
Maire gentil, raison veult que a present
L'acte excellent, dont tu as fait present,
Soit honnoré, exaulcé et levé
Ou lieu que doit chief d'œuvre estre eslevé :
Car le doulx myel que ta plume distille
Tant fort me plaist, que le tiens et dy stille
Plus elegant que d'orateur recent
Qui soit en cours, quant or en verray cent :
Et sans mentir ton œuvre manifeste
Le tien scavoir. C'est ce qui m'en infeste
A cry publicq dire que a ton merite
Est deu loyer qui scavant homme herite ${ }^{7}$.

Certes, la gloire littéraire est un lieu commun humaniste et y recourir dans le contexte d'une lettre de compliment est loin d'être l'apanage des poètes Français ${ }^{8}$, cependant il faut souligner que c'est un compatriote qui milite en faveur du vernaculaire et qui exalte la dynastie royale française (éventuellement l'histoire «nationale » dans ses Illustrations de Gaule [1509-13]) que Cretin ose placer au-dessus de tous, demandant au public de reconnaître son mérite et de lui payer son dû ${ }^{9}$. On retrouve le même souci d'inscrire les 
siens dans l'histoire à travers les épîtres que Robertet adresse à Chastelain, Bouchet à Parmentier, ou Molinet à Cretin.

Les Rhétoriqueurs n'opèrent pas de rupture radicale avec la tradition et reconduisent bon nombre de configurations et de thématiques héritées du Moyen Âge, composant par exemple des épîtres qui prennent la forme de ballades, comme celle adressée par Cretin à Christophe de Refuge, qui luy avoit demandé conseil de se marier ${ }^{10}$, ou de rondeaux, tel ce Responsif au même Refuge par la dame qui veut l'épouser ${ }^{11}$. Qu'il traite de casuistique amoureuse, de sujets historiques (Chronique françoise), de religion ou de morale (Chants royaux), Cretin s'inspire copieusement de ces prédécesseurs, de Guillaume de Machaut à Jean Froissart, jusqu'à Alain Chartier, et s'il incarne pour ses contemporains une certaine modernité, c'est d'abord et avant tout pour le raffinement extrême et la virtuosité des ses vers. En effet, il porte à son comble le travail de la forme, multipliant les rimes batelées, couronnées, équivoquées, voire doublement équivoquées, c'est-à-dire la reprise de sons identiques non seulement en fin de vers mais encore au début :

Filz, par escriptz j'ay veu qu'un jour a Han

Feiz pareilz cris que homme qui souffre ahan,

Portant le fes de guerre et ses alarmes.

Pourtant le fais qu'elle provocque a larmes

Tes doulx yeux secz, et sur eux l'eau tost rend,

Telz douze exces, plus souldain que torrent,

Laisse courir : son cours prandroit tes forces.

Les secourir est requis que t'efforces ${ }^{12}$.

On l'aura remarqué, cette épître à François Charbonnier privilégie les contraintes formelles au détriment du sens. Tout semble subordonné à la technique et on en déduit que, pour Cretin, le mérite esthétique se mesure à l'aune de la virtuosité. Il est vrai que son statut de poète pensionné par la cour ne laisse guère de place à la libre expression, d'où peut-être ce culte de la forme si marqué dans les poèmes de circonstances. L'auteur a beau prétexter qu'il écrit afin de « recreer d'aulcun petit plaisir ${ }^{13}$, ou « pour prendre esbat a passetemps honeste $»^{14}$, il y met tant d'application et de sérieux que le résultat dépasse le simple divertissement, surtout lorsque les acrobaties verbales s'étalent sur plusieurs pages. Certes, la recherche d'effets dérègle les mécanismes de l'expression et peut donner l'impression, comme l'affirmait naguère Zumthor ${ }^{15}$, que l'entreprise consiste à faire l'inventaire des potentialités de la langue, mais de véritables idéaux moraux et politiques s'incarnent à travers l'œuvre des Rhétoriqueurs et les épîtres de Cretin y font résonance.

C'est dans le chant royal, forme fixe héritée du Moyen Âge aux thèmes nobles et de style élevé, que l'on retrouve l'essentiel du discours idéologique 
de Cretin. Là, le fond l'emporte clairement sur la forme et il en émerge une vision d'un monde tourmenté, où le salut réside en Dieu et où la survie immédiate dépend du Prince, garant de la foi et de la paix contre les «Turcs maulditz et gens pleins d'insolence ${ }^{16}$. La Vierge y est beaucoup célébrée, dans la tradition de la poésie mariale ${ }^{17}$ et, fait à souligner, le pape y est vilipendé dans l'esprit du gallicanisme le plus orthodoxe. Après quelques pointes, Cretin adresse au saint siège cette mise en garde qui devient du coup le refrain de l'Invective contre la guerre papalle : " la loy permect se deffendre a l'espee ${ }^{18}$, même contre le souverain pontife, lorsque celui-ci croit pouvoir impunément « getter françois hors d'Italie » 19 et garder la main sur «les grans tresors du coffre apostolique $»^{20}$. Il y a de nombreux échos de ces chants royaux dans les épîtres. Ce sont des exhortations à «craindre Dieu, le servir et aymer ${ }^{21}$, des compliments échangées, des conseils prodigués aux pénitents, aux amis et même aux rois (il en a servi trois). Lorsqu'il parle de son maître ou s'adresse directement à lui, Cretin célèbre d'abord son mécène, n'hésitant pas à se plaindre d'ailleurs lorsqu'on lui coupe ses gages ${ }^{22}$, mais au panégyrique succède le rappel des devoirs et responsabilités du Prince :

\footnotetext{
Claire vertu, que l'en voit et qu'on oyt

Reluire en toy, porte augure et presage

Que immortel non obtiendras apres eage.

In facie legitur, si mes ditz

Sont averés, si je mentz ou mesdis

Le jugement en est tout manifeste

$[\ldots]$

Pour l'heure entens crier si undes graces

De mer flotant ont mis Angloys a terre,

Que a coups de lance et picque on les atterre ;

Qu'on charge acoup si frapper on y $\mathrm{a}^{23}$.
}

L'évocation de la guerre à mener n'a pas tant valeur d'injonction personnelle que d'exhortation générale et ne s'adresse pas tant au roi qu'au peuple : il s'agit d'exalter la figure du souverain protecteur de la foi et du peuple et de légitimer une fonction dont dépend, pour l'auteur, l'avenir même de la chrétienté, car : «tantost sera l'Eglise refformee, Estatz seront reduictz, sus et dessoubz ${ }^{24}$. Seul le Prince peut maintenir l'équilibre et assurer la pérennité de l'Église au sein d'une Europe en proie à l'hérésie et aux séditions. Ailleurs, dans une épître à Charbonnier, Cretin met en scène une allégorie du pouvoir autour des personnages de Gallus, Galathée et Pan qu'il explique ensuite en ces termes :

Tu entends bien, par le texte offrant ce,

Que Gallus prens pour le peuple de France, 
Galathea, ainsi que je l'applicque,

Est adaptee a la chose publique,

Et le pasteur nommé Pan, proprie

Ay cy endroit au Roy approprié.

Si dy qu'espoir des Françoys procureur

Apres ces temps divers nous procure heur ${ }^{25}$.

Le roi est ce pasteur qui protège le peuple contre les «loupz cerviers, ours et bestes sauvaiges » et qui procure espoir et réconfort en ces «temps divers ». Qui sont les bêtes sauvages de la fable ? L'auteur ne le précise pas, mais il est question d'un «traicté de paix » et donc de guerre. Il faut reconnaître ici que le Rhétoriqueur ne se contente pas simplement d'un jeu sur la forme : il sait mettre la rime et le mètre au service d'une idéologie politique cohérente ${ }^{26}$. En se faisant le propagandiste du régime, Cretin assume l'une des principales raisons d'être de sa charge : favoriser le maintien d'un ordre social. Le reste, après tout, n'est que vanité, car « les belles fleurs de Tulle, et rhetorique / N'ont point rendu exemptz d'exil mortel / Ceulx qu'ont instruictz ${ }^{27}$.

Conformiste affiché, le Rhétoriqueur sait aussi faire preuve d'ouverture face aux idées nouvelles. Sans souscrire aux tendances paganisantes du cicéronianisme - cela serait contraire à l'éthique de sa profession —, il semble reconnaître le mérite du parangon de l'éloquence (assez du moins pour faire rimer «Tulle » avec « cartule » ${ }^{28}$, c'est-à-dire lettre), et ses épîtres véhiculent les mêmes valeurs rhétoriques associées par lui et ses épigones à l'échange familier. On y remarque notamment une forte propension au retour de la pensée sur l'écriture, ces mouvements réflexifs caractéristiques du genre, impensables dans un cadre plus formel, où le sujet écrivant médite librement sur la nature du commerce épistolaire. Ainsi la correspondance renouvelle l'affection ${ }^{29}$, elle est une conversation avec un absent : «L'amy pensant a l'amy deviser ${ }^{30}$. Elle donne l'illusion d'une présence : « en visitant la lettre / Visiblement cuide avoir l'œil a l'aistre / De sa presence, et croit ouyr le son / Du sien parler ». Mais surtout, elle est le fruit de l'otium et d'un commerce privilégié : « amys privez / Cerchent plaisir du quel ne sont privez, / Car amplement et bien assez jouissent / De passetemps, dont souvent s'esjouissent ${ }^{31}$. Cretin exploite une topique épistolaire inspirée de l'Arpinate et il admet lui-même, dans une épître adressée à l'évêque de Glandeves, qu'il a voulu en faire son modèle, même s'il sait qu'il ne pourra jamais rivaliser d'éloquence avec lui :

[...] Tulle

Ne m'a laissé que bien petite part

Du doulx parler que a plusieurs gens depart,

Qui en tel art et stile trop mieulx se aydent 
Que je ne fays, et en scavoir m'excedent ;

Mais neantmoins si peu que en voy et scay,

J'ay bien voulu promptement faire essay

Vous presenter le labeur de ma muse,

Selon le train rural ou je me amuse

Si vous supply le recevoir de gré,

Comme s'il fust en plus parfaict degré

Et si a point, jour et heure oportune

Nouvelle en court, d'adventure en porte une $\mathrm{e}^{32}$.

L'épistolier entreprend donc de s'essayer « promptement» au genre illustré par lui en composant dans un style qu'il qualifie presque de rustique, et de fait moins sophistiqué qu'à l'ordinaire, une épître à un supérieur hiérarchique. On s'éloigne ici du formalisme de la rhétorique épistolaire médiévale, qui exigeait en de telles circonstances un registre élevé et une dispositio calquée sur celle de l'oratio. Mais, à la différence des Italiens ou de son compatriote Christophe de Longueil, Cretin n'opte pas pour une imitation servile de Cicéron et il se contente d'adapter à l'épître en vers les motifs et l'axiologie de la lettre familière en prose.

«L'humanisme» de Cretin se manifeste essentiellement à travers les lieux éthiques et pathétiques qu'il emploie. On a montré ailleurs qu'une rhétorique humaniste de l'épistolaire est en voie de constitution à l'époque et qu'elle se caractérise notamment par l'exploitation d'un éthos informel ainsi que par le recours à une topique des devoirs de l'amitié inspirée par la redécouverte des œuvres de Cicéron ${ }^{33}$. Or les épîtres de Cretin sont remplies de considérations sur l'amitié, de témoignages d'affection et de bienveillance : «Mon bon amy se faire te scavoye / Aulcun plaisir, cela s'en va sa voie/ Je le ferois et de bien bon courage $»^{34}$; «Et, si tu veulx, apres mort et en vie / Toujours aurons de nous aymer envye » 35 ; «Faiz que nos cueurs ensemble soient tassez, / Et se as papier ou ancre teincte en voye, / Replique ad ce que ouan Cretin t'envoye ! ${ }^{36}$. L'amitié que Cretin exalte est, à l'exemple des anciens, fondée sur la vertu et sur la réciprocité. Il se plait à évoquer les règles de civilité et ses commentaires ont parfois même un caractère prescriptif :

Le bon ami doibt expres regarder

De reciproque amour l'ordre garder

Et tout ainsi que plaisir faict, requiert

Aultre plaisir, l'amy grant erre quiert,

Et doibt querir, au sien amy complaire,

En ce qu'il scet que a luy peult beaucoup plaire ${ }^{37}$.

En fait, ces considérations qui interviennent si souvent dans les correspondances familières visent à manifester ou à évaluer le conformisme socio-dis- 
cursif des interlocuteurs, c'est-à-dire leur adhésion à des valeurs communes. La mise en scène de l'affectivité sert à établir l'identité des images réciproques, lesquelles se définissent à travers l'écriture et au gré de l'échange. Elle peut servir à souligner les différences ou au contraire à les gommer. Si les épistoliers veulent maintenir le contact, ils doivent négocier ces différences pour arriver à un minimum d'identité de vues et de sentiments. Pour que la relation perdure, il faut l'entretenir et le commerce épistolaire est particulièrement propice aux demandes de faveurs et aux offres de service : «Donques a Paris l'epistole receue, / Et du porteur bien au vray l'erre sceue / De son retour, vous plaise a coup mander / Vos bons plaisirs, et les me commander ${ }^{38}$. Cette sollicitude, malgré son caractère conventionnel, contribue à établir un rapport de proximité qui autorise une expression plus souple, et l'on s'étonne de retrouver chez l'équilibriste du mètre et de la rime des épîtres plus informelles :

J'espere aussi, qu'en lisant ceste epistre
Ton franc vouloir et liberal arbitre
Prendront en eulx deliberation -
Sur ce n'ayans consideracion
Au rudde stille et mauplaisant langage,
Mais au desir qu' ores te baille en gage-
Incontinent mectre la plume en main
Pour m'envoyer, ung jour apres demain,
Du tien parler la doulce confiture ${ }^{39}$.

Le ravalement de son style relève bien sûr du topos de modestie, figure imposée qui ne leurre personne, cependant il s'accompagne à l'occasion, comme c'est le cas ici, d'un relâchement de la forme qui situe l'échange dans un registre moins « oratoire » et plus près du mode familier. Même la dispositio tend à s'assouplir : l'absence de salutations, la brièveté des formules de valédiction, sont autant d'éléments qui, avec la mise en scène de la benevolentiae caritatem, contribuent à rapprocher la pratique épistolaire de Cretin de celle de ses contemporains.

L'œuvre de Guillaume Cretin participe, comme celle de ses confrères Jean Lemaire de Belges (1473-1520) et Jean Bouchet (1476-1558), de l'affirmation d'une identité culturelle et c'est dans cette perspective qu'il convient d'apprécier son traditionalisme et son adhésion sélective aux modèles rhétoriques de l'époque. Écrivains pensionnés des Princes, les Rhétoriqueurs avaient pour tâche première d'en soutenir les politiques, aussi ils n'hésitent pas à intervenir dans les débats qui les opposent à Rome et pour faire la propagande du gallicanisme. Mais au-delà de cette finalité qui les mobilise tous, il faut reconnaître que certains ont su, tel Guillaume Cretin dans ses épîtres, faire leur miel du renouveau de la rhétorique tout en 
préservant les formes et les traditions françaises. C'est pourquoi il conviendrait de relativiser l'interprétation du propagandisme des Rhétoriqueurs ou de leur conservatisme affiché et de parler plutôt, quand vient le temps de les situer par rapport aux courants contemporains, d'un humanisme dissident.

\section{Université du Québec à Chicoutimi}

\section{Notes}

1. Voir notamment Janine Basso, «Les traductions en français de la littérature épistolaire italienne aux XVI ${ }^{\mathrm{e}}$ et XVII ${ }^{\mathrm{e}}$ siècles », Revue d'histoire littéraire de la France 78.3 (1978) : 906-18.

2. Voir François Lecercle, «Un pétrarquisme épistolaire : les Lettres amoureuses d'Étienne du Tronchet », dans La littérature de la Renaissance, éd. Marguerite Soulié (Genève, Slatkine, 1984), p. 213-25.

3. Claude La Charité rappelle que « l'épître a été constitué en genre poétique à part entière et privilégié par les Grands Rhétoriqueurs » (La rhétorique épistolaire de Rabelais [Québec, Éditions Nota Bene, 2003], p. 71). L'auteur souligne par ailleurs que « contrairement au XVII ${ }^{\mathrm{e}}$ siècle, la Renaissance ne différencie pas la lettre de l'épître sur la seule base de la facture prosaïque ou versifiée » (p. 70). En fait, le terme " épître », issu du latin epistola, lui-même emprunté au grec epistole (message), désigne d'abord toute forme de lettres, puis, à partir du XII ${ }^{\mathrm{e}}$ siècle, celles écrites par les Apôtres. Au XVI ${ }^{\mathrm{e}}$ siècle, il tend à prendre le sens de « lettre en vers », chez les Rhétoriqueurs notamment, mais on l'emploie encore au sens large (Hélisenne de Crenne). Si la lettre missive (du latin missus : envoyée) est d'abord celle destinée à un correspondant officiel (à partir de 1465), elle passe dans l'usage courant au sens général de «lettre » vers la fin du XVI ${ }^{\mathrm{e}}$ siècle.

4. Anna Rigamonti souligne la modernité de la poésie des Rhétoriqueurs et décrit leur effort pour « élever la langue vulgaire au niveau de la langue nationale, de la langue digne d'être comparée par sa richesse à celle qui a fait la gloire de Rome » dans son article « Guillaume Cretin : la contribution d'un rhétoriqueur à l'enrichissement du français », Moyen français 41 (1996) : 431

5. Clément Marot, Euvres poétiques, éd. Gérard Defaux, 2 vol., «Classiques Garnier» (Paris, Bordas, 1993), $2: 361$.

6. Euvres poétiques de Guillaume Cretin, éd. Kathleen Chesney (Genève, Slatkine Reprints, 1977). Toutes les citations de Guillaume Cretin proviennent de cette édition.

7. A son amy Le Maire Cretin mande Salutz assez pour comble un mande, p. 338-39.

8. Voir Françoise Joukovsky, La gloire dans la poésie française et néolatine du XVIe siècle des rhétoriqueurs à Agrippa d'Aubigné, « Travaux d'humanisme et Renaissance »(Genève, Droz, 1969).

9. Lemaire de Belges rapporte que c'est Cretin qui l'encouragea à « mettre la main à escrire en ceste nostre langue françoise et gallicane » (cité par Henri Chamard, Les origines de la poésie française de la Renaissance [Paris, Boccard, 1920], p. 158).

10. Dudict Cretin a Christofle de Refuge, Maistre d'Hostel de Monseigneur d'Alençon, p. 290.

11. Ce responsif a été repris par Rabelais au chapitre XXI du Tiers livre. Il en fait la réponse du vieux poète Raminagrobis au dilemme de Panurge.

12. Dudict Cretin audict Charbonnier, p. 275. 
13 Ledit Cretin a Macé de Villebresme, Valé de Chambre du Roy, p. 259.

14. Dudict Cretin audict Frere Jehan Martin, p. 304.

15. Paul Zumthor, Le masque et la lumière (Paris, Éditions du Seuil, 1978), p. 54-55.

16. Chant royal VII, p. 17.

17. Sur la poésie mariale dans l'œuvre des Rhétoriqueurs, voir Anne Berthelot, « De Gautier de Coinci aux Rhétoriqueurs : glossolalie et poésie mariale », Moyen français 39 (1996) : 25-37.

18. Invective contre la guerre papalle, p. 58-59.

19. Ibid.

20. Ibid.

21. Epistre aux Filles Penitentes, p. 320.

22. Dudit Cretin au roy François Premier et present, pour ce que l'on luy avoit dit qu'il estoit cassé de ses gaiges, p. 227.

23. Dudict Cretin a monseigneur duc de Valoys, conte d'Angoulesme, a present roy, p. 225-26.

24. Autre epistre dudit Cretin audit Charbonnier, p. 289.

25. Ibid., p. 288.

26. Dans un article intitulé «Poésie contre(-)éloquence. Destitution en règles du poète courtisan », Moyen Français 43 (1998) : 99-121, Mawy Bouchard rappelle que le métier ne consiste pas seulement à faire des rimes : «Le Rhétoriqueur sous les règnes de Charles VII à Louis XII accompagne le roi dans ses années de formation, en tant que précepteur ; dans ses fonctions de dirigeant, en tant que secrétaire, conseiller et diplomate ; dans ses fonctions de représentant officiel, en tant qu'auteur de pièces adaptées aux circonstances. "Versifier ressortit à leur office", certes, mais leur office est varié » (p. 100). Sur les Rhétoriqueurs propagandistes, voir Cynthia Jane Brown, The Shaping of History and Poetry in Late Medieval France : Propaganda and Artistic Expression in the Works of the Rhétoriqueurs (Birmingham, AL, Summa Publications, 1985).

27. Translation du chant de Misere faict par ledict Cretin, p. 313.

28. Dudict Cretin a Monseigneur l'Evesque de Glandeves, p. 297.

29. «Quant poste arrive, et porte erre nouvelle, /L'affection, sans doubter, renouvelle ( Dudict Cretin a Monseigneur l'Evesque de Glandeves, p. 297).

30. Ibid.

31. Ibid.

32. Ibid.

33. Voir Luc Vaillancourt, La lettre familière au XVIe siècle. Rhétorique humaniste de l'épistolaire (Paris, Champion, 2003).

34. Dudict Cretin a Jacques de Bigue Varlet de Chambre des Rois Lois Douziesme, Charles Huictiesme, et François Premier, p. 255.

35. À Maistre François Charbonnier, lors secretaire de mon seigneur le Duc de Valois, Conte d'Angolesme, a present Roy, p. 273.

36. Dudict Cretin a Honorat de la Jaille Escuyer de Monseigneur le Duc d'Alençon, p. 267.

37. Autre epistre dudit Cretin audit Charbonnier, p. 283.

38. Dudict Cretin a Monseigneur l'Evesque de Glandeves, p. 298.

39. Ledit Cretin a une dame lyonnoise, p. 293. 